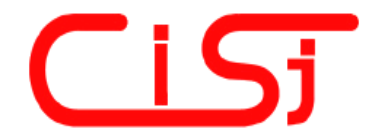

\title{
SIMULATION OF DC-DC CONVERTER SYMBOLIC DYNAMICS: DETECTION AND IDENTIFICATION OF BIFURCATION TYPES
}

\author{
Abdelaziz Hamzaoui ${ }^{1)}$, Pavel Ustinov ${ }^{2)}$, Andrey Sholonik ${ }^{3)}$, Yury Kolokolov ${ }^{4)}$ \\ 1) LAM - UFR Sciences Exactes et Naturelles - Moulin de la Housse \\ BP 1039 - 51687 REIMS Cedex 2 - France \\ Tel: +33 (0) 3254246 43, Fax: +33 (0) 326913106 \\ e-mail: a.hamzaoui@iut-troyes.univ-reims.fr \\ 2,3,4) Department of Design and Technology of Electronic and Computer Systems, \\ Orel State Technical University, \\ 29 Naugorskoye Shosse, 302020 Orel, Russia \\ Tel: ${ }^{2,3)}+70862419879,{ }^{4)}+70862421661$, Fax: ${ }^{2,3,4)}+70862416684$ \\ e-mail: ${ }^{2)}$ p_s_ustinov@orel.ru, ${ }^{3)}$ sholonik@ostu.ru, ${ }^{4)}$ kolokolov@ostu.ru
}

\begin{abstract}
In this paper continuous-time model to discrete-time symbolic model transformation for an algorithmization of the DC-DC buck converter dynamics investigation process is derived. The proposed concept of symbolic index allows evaluate quantitatively the symbolic model change and makes possible the "nonsmooth" bifurcations revealing. The numerical simulation that had been carried out verifies utilizing efficiency of symbolic modeling both as for possible bifurcation pattern forecasting, as for bifurcation type identification.
\end{abstract}

Keywords: simulation, DC-DC buck converter, symbolic model, identification, bifurcation.

\section{INTRODUCTION}

Application and further development of the pulse energy conversion systems (PECS) is one of the main trends of energy generation, conversion and distribution process efficiency increasing. Switching controller is "the heart" of any PECS. In most practical cases the required stable operating regime of switching controller is an operation at the fundamental frequency (period-1 operation) $[1,2]$. The fundamental operation refers to an operating regime, when the output voltage and current waveforms repeat at the same rate as the driving clock.

In this paper the DC-DC buck converter model based on symbolic dynamics (symbolic models) is considered. This converter refers to the class of dynamical systems with discontinuous nonlinearities [3]. Strong nonlinearity, as a result of the switching action [4], and a wide range of operating parameter values (for instance, the controller feedback gain and/or load impedance) changing are the basic properties of the PECS. These properties make possible an appearance of undesirable dynamic modes during switching controller operation, resulting in essential degradation of the output energy quality. Any of subharmonic, quasi-periodic and chaotic modes is regarded as undesirable $[2,5]$ and should be avoided at the design stage. An importance of undesirable modes elimination, in turn, gives rise to a problem of the buck converter dynamics simulation for the purpose of emergency situations forecasting, detection and insulation [6].

Since dynamics investigation process is rich in awkwardness of the required mathematical operations, a desire to simplify this research is inherent (especially for practitioners). One can realize it with the help of dynamic mode symbolic description [3, 7, 8]. Symbolic dynamics is a powerful mathematical tool that describes system evolution by means of symbol set and lets to formalize for further algorithmization and simplify significantly for understanding dynamics investigation process $[3,9]$.

Generally speaking, there are two bifurcation types in the system dynamics: "smooth" (for instance, period doubling bifurcation, saddle-node and Hopf bifurcation, etc.) $[4,10,11]$ and "nonsmooth" (for instance, transition of period-1 to chaos, transition of period-2 to period-3, etc.) $[4,10$, $12,13]$.

In this paper continuous-time model to discretetime symbolic model transformation had been 
realized for an algorithmization of the dynamics investigation process. The introduced concept of the symbolic index (based on symbolic dynamics) is proposed to apply for quantitative estimation of the symbolic model change and for the efficient identification of "nonsmooth" bifurcations.

\section{MATHEMATICAL MODEL}

Equivalent scheme of the buck converter is shown in Fig. 1.

Mathematical model of the power unit of the buck converter, corresponding to the equivalent scheme (Fig. 1), has the form

$$
\begin{aligned}
& \frac{d \mathbf{X}(\gamma)}{d \gamma}=T\left(\mathbf{A} \cdot K_{F 1}+\mathbf{A}_{1} \cdot\left(1-K_{F 1}\right)\right) \mathbf{X}(\gamma)+ \\
& +T \cdot \mathbf{B} \cdot K_{F 0},
\end{aligned}
$$

where $\mathbf{X}(\gamma)=\left(\begin{array}{l}i(\gamma) \\ u(\gamma)\end{array}\right)$ is state variable vector; $i(\gamma)$ is the inductor current; $u(\gamma)$ is the capacitor voltage; $\gamma=\frac{t}{T}, \gamma \in[0,1]$ is the relative pulse duration; $T$ is the pulse-width modulation (PWM) clock instant;

$$
\mathbf{A}=\left(\begin{array}{cc}
-\frac{1}{L}\left(R_{1}+\frac{R_{2} \cdot R_{3}}{R_{2}+R_{3}}\right) & -\frac{R_{3}}{L \cdot\left(R_{2}+R_{3}\right)} \\
\frac{R_{3}}{C \cdot\left(R_{2}+R_{3}\right)} & -\frac{1}{C \cdot\left(R_{2}+R_{3}\right)}
\end{array}\right),
$$

$\mathbf{A}_{1}=\left(\begin{array}{cc}0 & 0 \\ 0 & -\frac{1}{C \cdot\left(R_{2}+R_{3}\right)}\end{array}\right), \quad \mathbf{B}=\left(\begin{array}{c}\frac{E}{L} \\ 0\end{array}\right)$ are the

square matrices and column vector, determined by element values of the equivalent scheme in Fig.1.

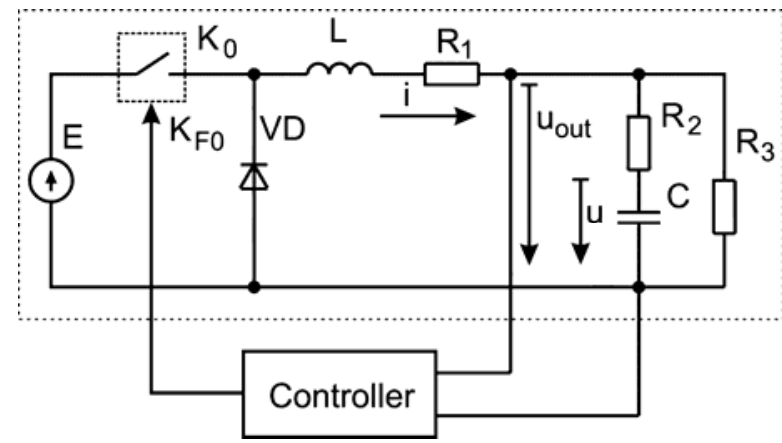

Fig. 1 - Equivalent circuit of the buck converter.

Controller realizes trailing edge pulse-width modulation and proportional control of the output voltage. The pulse function $K_{F 0}$ in model (1) is calculated according to the algorithm

$$
K_{F 0}= \begin{cases}1, & 0<\gamma \leq \gamma_{0} \\ 0, & \gamma_{0}<\gamma \leq 1\end{cases}
$$

where $\gamma_{0}$ is the switch moment, corresponding to transition of $\mathrm{K}_{0}$ switch to non-conducting state and diode VD to conducting state.

Value $\gamma_{0} \in[0,1]$ is evaluated as the least root of the equation, which determines a surface where the right-hand part of (1) has discontinuities

$$
\begin{aligned}
& \zeta_{0}(\mathbf{X}(\gamma), \gamma)=\alpha \cdot\left(U_{r e f}-\beta \cdot \mathbf{C}_{\mathbf{0}} \cdot \mathbf{X}(\gamma)\right)- \\
& -U_{0} \cdot \gamma=0,
\end{aligned}
$$

where $\alpha$ is the proportional feedback gain; $\beta$ is the gain of the output voltage sensor; $U_{\text {ref }}$ is the reference voltage; $U_{0}$ is the sawtooth voltage amplitude; $\mathbf{C}_{\mathbf{0}}$ is the row vector which sets up a correspondence between $\mathbf{X}(\gamma)$ and the voltage value of the controller input $\left(u_{\text {out }}\right)$.

The pulse function $K_{F l}$ in model (1) is calculated according to the algorithm

$$
K_{F 1}= \begin{cases}1, & \gamma_{0}<\gamma \leq \gamma_{1} \\ 0, & \gamma_{1}<\gamma \leq 1\end{cases}
$$

where $\gamma_{1}$ is the switch moment, corresponding to transition of diode VD to non-conducting state.

Value $\gamma_{1} \in\left[\gamma_{0}, 1\right]$ is evaluated as the least root of the equation

$$
\zeta_{1}(\mathbf{X}(\gamma))=\mathbf{C}_{1} \cdot \mathbf{X}(\gamma)=0, \mathbf{C}_{1}=(1
$$

\section{ALGORITHMIZATION OF THE BUCK CONVERTER DYNAMICS INVESTIGATION PROCESS}

For the further development of the algorithmization procedure of the buck converter dynamics investigation process it is necessary to introduce some definitions for the clarity of discussion.

Let $\mathbf{G}$ to be considered a state space of the buck converter mathematical model (1-5). There are three $\mathbf{G}_{\mathbf{1}}, \mathbf{G}_{\mathbf{2}}, \mathbf{G}_{\mathbf{3}}$ domains in $\mathbf{G}$ space that can be determined according to the values of pulse functions $K_{F 0}, K_{F l} . \mathbf{S}_{\mathbf{1}}, \mathbf{S}_{\mathbf{2}}, \mathbf{S}_{\mathbf{3}}$ surfaces are the boundaries of the $\mathbf{G}_{\mathbf{1}}, \mathbf{G}_{\mathbf{2}}, \mathbf{G}_{\mathbf{3}}$ domains. $\mathbf{S}_{\mathbf{2}}$ surface is divided into two components $\mathbf{S}_{2(1)}$ and $\mathbf{S}_{2(2)}$ additionally (for the detailed research of the state space geometrical structure see [7]).

Definition: A section of the trajectory that is placed between two surfaces, on which system 
structure does not change, is called the simplest section $[7,8]$.

Definition: The simplest trajectory is a sequential combination of a few simplest sections, the start and end points of which belong to neighbor $\mathbf{S}_{\mathbf{2}}$ surfaces [7, 8].

The possible eleven types of simplest trajectories correspond to the buck converter model with PWM (1-5). These trajectories are summarized in Table 1.

The sequential notation of the indices of simplest sections, that compose the simplest trajectory, is applied for notation of the simplest trajectory symbolic model $[3,7,8]$. These indices are separated by dot.

Definition: The notation $\left(a_{1}, a_{2}, \ldots a_{k}\right)$, where $a_{1}$, $a_{2}, \ldots a_{k}$ - the numbers of trajectories (see Table 1) that are evaluated with usage of the simplest trajectory identification algorithm [8], $\mathrm{k}$ - the dynamic mode periodicity, is considered to be the symbolic model of the buck converter dynamic mode.

Table 1 - Simplest trajectories of the buck converter model

\begin{tabular}{|c|l|c|c|}
\hline Number & \multicolumn{1}{|c|}{ Trajectory } & $\gamma_{0}$ & $\gamma_{1}$ \\
\hline 1 & $2(1) 2(1)$ & 1 & 0 \\
\hline 2 & $2(1) 1.12(1)$ & $\mathrm{z}_{1}$ & $1-\mathrm{z}_{1}$ \\
3 & $2(1) 1.12(2)$ & $\mathrm{z}_{1}$ & $1-\mathrm{z}_{1}$ \\
\hline 4 & $2(1) 1.13 .32(1)$ & $\mathrm{z}_{1}$ & $\mathrm{z}_{2}$ \\
5 & $2(1) 1.13 .33$ & $\mathrm{z}_{1}$ & $\mathrm{z}_{2}$ \\
\hline 6 & $2(2) 2(2)$ & 0 & 1 \\
7 & $2(2) 2(1)$ & 0 & 1 \\
\hline 8 & $2(2) 3.32(1)$ & 0 & $\mathrm{z}_{2}$ \\
9 & $2(2) 3.33$ & 0 & $\mathrm{z}_{2}$ \\
\hline 10 & $32(1)$ & 0 & 0 \\
11 & 33 & 0 & 0 \\
\hline
\end{tabular}

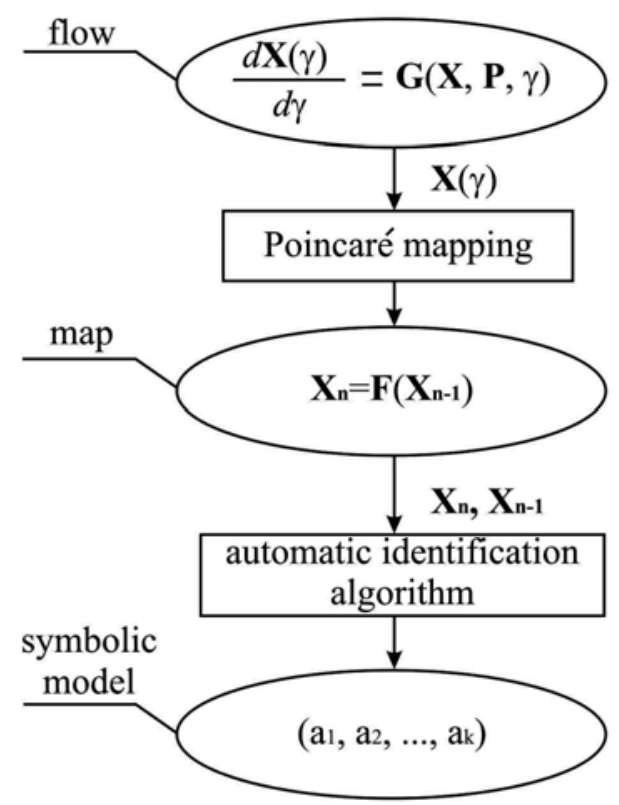

Here $z_{1}, z_{2}$ - arbitrary real values within the interval $[0 ; 1]$.

Now it is possible to discuss the algorithmization procedure directly.

The buck converter mathematical model (1) represents by itself a system of ordinary differential equations of second order with the discontinuous right-hand part. The time in this model is considered to be the continuous value, and that is why such models are called the flows in nonlinear dynamics.

The investigation of these systems, as mentioned above, is accompanied with significant computational difficulties because of the essential nonlinearity presence. Therefore the discrete-time models, so called the mappings, are utilized for this class of systems investigation. In most practical cases the Poincare stroboscopic mapping is applied and considered to be an exact investigation method of highly nonlinear systems

$$
\mathbf{X}_{\mathrm{n}}=\mathbf{F}\left(\mathbf{X}_{\mathbf{n}-1}\right)
$$

where $\mathbf{X}_{\mathbf{n}-1}$ and $\mathbf{X}_{\mathbf{n}}$ are the state variable vectors at time moments $t_{n-1}=(n-1) \cdot T$ and $t_{n}=n \cdot T$, respectively; $T$ is the PWM clock instant; $n \in \mathbb{N}$ is the number of PWM clock instant.

The results of discrete-time modeling are utilized as the input data of simplest trajectory identification algorithm [8] for dynamic mode symbolic models composition.

The noted previously algorithmization procedure of the buck converter dynamics investigation is shown in Fig. 2.

Fig. 2 - The general scheme of the flow to symbolic model transformation algorithm. 


\section{SYMBOLIC INDEX UTILIZING FOR THE BIFURCATION DETECTION AND THEIR TYPE IDENTIFICATION}

The specific symbolic model $\left(a_{1}, a_{2}, \ldots a_{n}\right)$ (see Section 3) corresponds to mapping point motion in the phase space and maps the simplest trajectory sequence that passed by mapping point. Border collision bifurcations occur, when the number of simplest sections (from which the simplest trajectory consists) that mapping point passes, changes [3]. Therefore, symbolic model changes and this change can be estimated quantitatively, when border collision bifurcation occurs. The quantitative estimation of this change can be calculated with the help of symbolic index.

Definition: Symbolic index is an average simplest trajectory number in the considered parameter space interval (or at the point of the parameter space), which gives a quantitative estimation of simplest trajectory contribution to system dynamics and is evaluated as

$$
N=\frac{\sum_{i=1}^{K} N_{i}}{K},
$$

where $N_{i}$ is the trajectory number at the each clock instant (Table 1); number of clock instants $K$ is calculated according to the algorithm

$$
K=\left\{\begin{array}{l}
\frac{\alpha_{2}-\alpha_{1}}{\Delta \alpha} \cdot K_{1}, \quad \alpha_{1} \neq \alpha_{2} ; \\
K_{1}, \quad \alpha_{1}=\alpha_{2} ; \quad \alpha_{1}, \alpha_{2} \in \mathbf{P} ;
\end{array}\right.
$$

where $\alpha_{1}, \alpha_{2}$ are the lower and upper borders of considered parameter space interval $\left[\alpha_{1} ; \alpha_{2}\right] \in \mathbf{P} ; \mathbf{P}$ is the model (1) parameter space; $\Delta \alpha$ is the step of parameter $\alpha$ changing; $K_{1}=$ const is the clock instant number, calculated for each parameter $\alpha$ value.

The theorem below may be stated according to previously mentioned facts.

Theorem: Let $\alpha \in \mathbf{P}$ (P is the parameter space), $N_{-}, N_{+}, T_{-}, T_{+}$are symbolic indices and output waveform periods for $\alpha=\alpha_{\text {bif }}-\varepsilon$ and for $\alpha=\alpha_{\text {bif }}+\varepsilon \quad(\forall \varepsilon>0)$ respectively. Thus, if $N_{\text {- }}$ $\neq N_{+}$, then "nonsmooth" bifurcation had occurred at the point $\alpha=\alpha_{b i f}$, else, if $N_{-}=N_{+}$and $T_{-} \neq T_{+}$, then "smooth" bifurcation had occurred.

Topological structure of the phase space changes, when system undergoes border collision, and results in symbolic model, and, correspondingly, symbolic index change. Thus, symbolic index change implies border collision bifurcation.

\section{NUMERICAL SIMULATION}

The purpose of numerical simulation is an efficiency confirmation of the proposed algorithm and symbolic index concept application for the PECS dynamics investigation, in general, and for bifurcation type identification, in particular.

The numerical simulations were carried out on the PWM voltage-controlled DC-DC buck converter with the proportional feedback gain.

The values of the buck converter elements during simulation process were the following: $R_{1}=0,27 \Omega$; $R_{2}=0,18 \Omega ; \quad R_{3}=16,9 \Omega ; \quad L=8,9 \cdot 10^{-4} \mathrm{H} ;$ $C=1,7 \cdot 10^{-4} \mathrm{~F} ; \quad E=24 \mathrm{~V} ; \quad \beta=1 / 4 ; \quad U_{0}=6 \mathrm{~V} ;$ $U_{\text {ref }}=3 \mathrm{~V} ; \quad f=8,63 \cdot 10^{3} \mathrm{~Hz}$. The proportional feedback gain $\alpha$ was varied within the interval $[0 ; 165]$.

The bifurcation diagram (Fig. 3) is divided into 3 domains: $\mathbf{A}, \mathbf{B}$ and $\mathbf{C}$. The time diagrams in each domain are depicted in Figures 4, 5 and 6 respectively. At the each point of the A domain the value of symbolic index is $N=2$, the value of the output waveform period is $T=1$; in the $\mathbf{B}$ domain the value of symbolic index is $N=2$, the value of the output waveform period is $T=2$; in the $\mathbf{C}$ domain the value of symbolic index is $N=3$, the value of the output waveform period is $T=2$. Thus, according to the theorem (see, for details, Section 4), at the point $\alpha=\alpha_{b i f 1}$ the standard "smooth" period doubling bifurcation had occurred, and at the point $\alpha=\alpha_{b i f 2}$ the "nonsmooth" border collision bifurcation had occurred. Changes of the output waveforms are demonstrated in the time diagrams (Figures 4, 5, 6): for instance, one can clearly see period doubling in Fig. 5 and appearance of the zero-current section (so called discontinuous conduction mode, DCM) in Fig. 6.

There are the following quantities in the Fig. 3: $\gamma_{0}$ is the relative duration of $K_{0}$ switch conducting state; $\alpha$ is the proportional feedback gain of the switching controller.

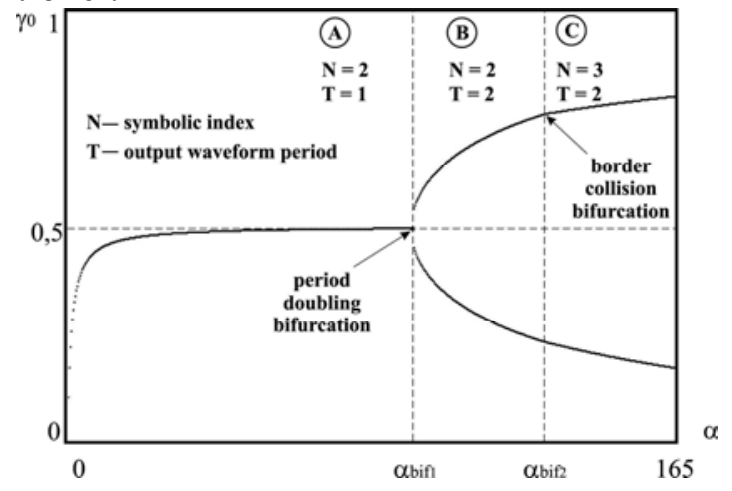

Fig. 3 - Numerically simulated bifurcation diagram. 


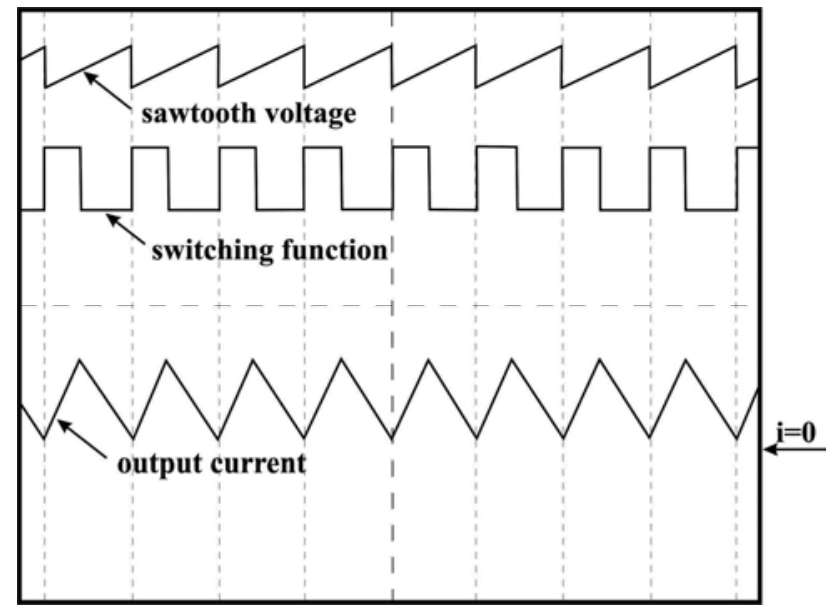

Fig. 4 - Numerically simulated time diagram in the A domain (period-1).

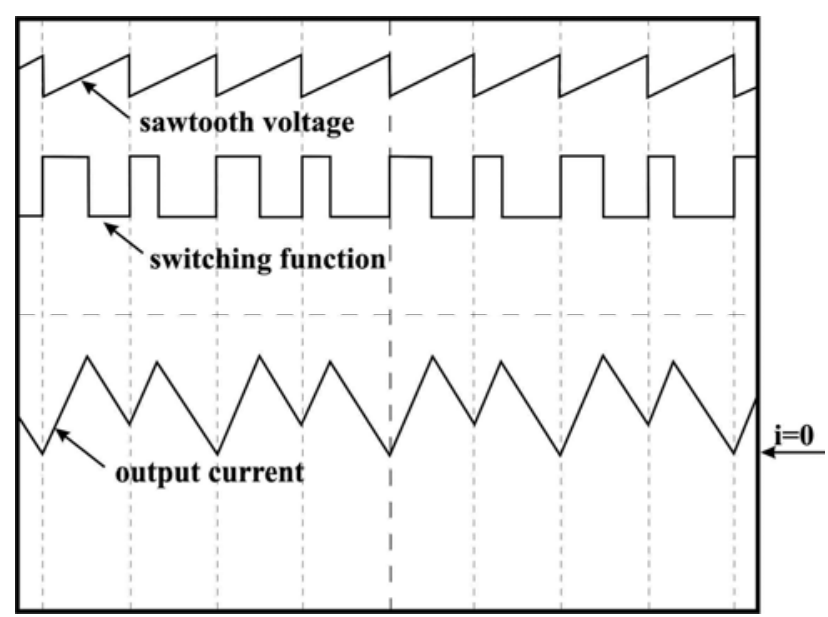

Fig. 5 - Numerically simulated time diagram in the B domain (period-2).

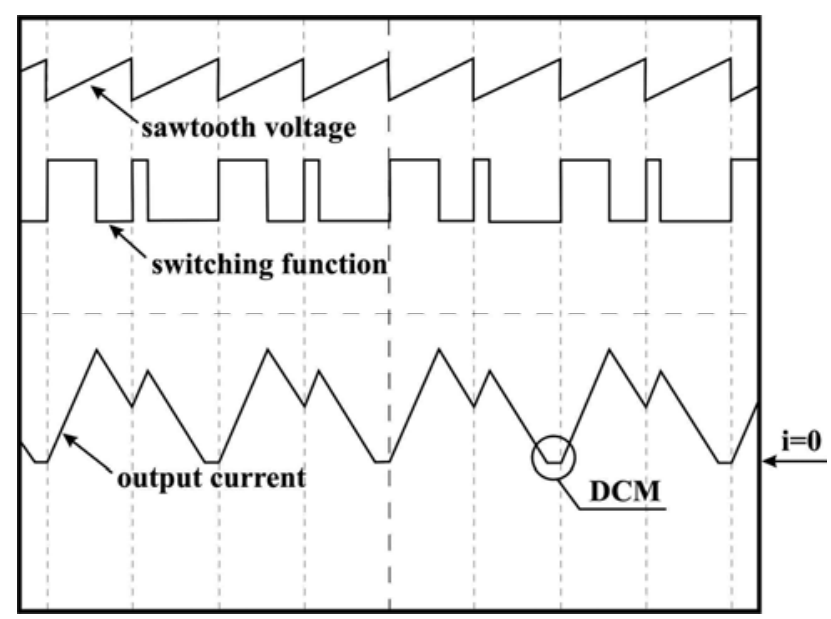

Fig. 6 - Numerically simulated time diagram in the $C$ domain (period-2, DCM).

\section{CONCLUSION}

A significant interest rising to perspective methodology of the symbolic model composition and its utilizing for the essential nonlinear systems dynamics investigation has been observed over the past decade.

Symbolic modeling looks to be the most promising as it offers the formalization and, thus, the automation of dynamics investigation process. Forecasting of undesirable phenomena appearance, which can lead to the emergency situations, and these phenomena elimination already at the design stage becomes possible. Moreover, symbolic modeling simplifies understanding of possible bifurcations general pattern and, in particular, the mechanism of bifurcation appearance.

In this paper symbolic index concept that was introduced in the framework of above-mentioned methodology, is calculated on base of the developed flow to symbolic model transformation algorithm and lets to reveal efficiently the specific nonlinear phenomena, which are generic for "nonsmooth" systems only (so called "nonsmooth" bifurcations), with help of the quantitative estimation.

\section{REFERENCES}

[1] C.K. Tse, Y.M. Lai and M.H.L. Chow, "Control of Bifurcation in DC/DC Converters: An Alternative Viewpoint of Ramp Compensation," International Conference on Industrial Electronics, Control and Instrumentation (IECON'2000), Nagoya, Japan, pp. 2413-2418, October 2000.

[2] C.K. Tse and Y.M. Lai, "Controlling Bifurcation in Power Electronics: A Conventional Practice Re-visited," Latin American Applied Research, vol. 31, pp. 177-184, September 2001.

[3] M.I. Feigin, Forced Oscillations in Systems with Discontinuous Nonlinearities. Moscow: Nauka, 1994 (in Russian).

[4] G. Olivar, C. Batlle, A. Miralles, I. Massana, Y. Zuniga, "Bifurcation Phenomena in a Sliding-Mode Controlled Boost Converter," Proc. of the $10^{\text {th }}$ Power Electronics and Motion Control Conference (EPE-PEMC'2002), Cavtat \& Dubrovnik, Croatia, September 2002. (CD-ROM) file ssin-05.pdf.

[5] J. Deane, D. Hamil, "Instability, Subharmonics and Chaos in Power Electronic Systems," IEEE Transactions on Power Electronics, vol. 5, no. 3, pp. 260-268, 1990.

[6] Yu.V. Kolokolov, S.L. Koschinsky, K.H. Adjallah, "The Mathematical Problems of Forecasting Adequacy of Emergency Situations in the Dynamics of the Pulse Energy Conversion Systems When Using Bifurcation Approach," Proc. of the Second International Conference on 
Mathematical Methods in Reliability (MMR'2000), Bordeaux, France, pp. 603-606, July 2000.

[7] S.L. Koschinsky, A.P. Sholonik, P.S. Ustinov, "Symbolic Models of the Pulse Energy Conversion Systems Dynamics," International Scientific Journal of Computing, vol. 2(1), pp. 73$78,2003$.

[8] Yu.V. Kolokolov, S.L. Koschinsky, A.P. Sholonik, P.S. Ustinov, "Symbolic Model Based Identification of Pulse System Dynamics," Proceedings of the $3^{\text {rd }}$ International Conference on Neural Networks and Artificial Intelligence (ICNNAI'2003), Minsk, Belarus, pp. 215-219, November 2003.

[9] D. Lind and B. Marcus, An Introduction to Symbolic Dynamics and Coding. New York, USA: Cambridge Univ. Press, 1995.

[10] C.K. Tse and M. di Bernardo, "Complex Behavior in Switching Power Converters," Proceedings of IEEE, Special Issue on Applications of Nonlinear Dynamics to Electronic and Information Engineering, vol. 90, no. 5, pp. 768781, May 2002.

[11] C.C. Fang, E.H. Abed, "Local Bifurcations in DC-DC Converters," Proc. of the $10^{\text {th }}$ Power Electronics and Motion Control Conference (EPE-PEMC'2002), Cavtat \& Dubrovnik, Croatia, September 2002. (CD-ROM) file ssin-02.pdf.

[12] S. Banerjee, P. Ranjan, and C. Grebogi, "Bifurcation in Two-Dimensional Piecewise Smooth Maps - Theory and Applications in Switching Circuits," IEEE Trans. Circuits Syst. I, vol. 47, pp. 633 - 643, May 2000.

[13] S. Banerjee, E. Ott, J.A. Yorke, and G.H. Yuan, "Anomalous Bifurcation in dc-dc Converters: Borderline Collisions in Piecewise Smooth Maps," in Proc. IEEE Power Electronics Specialists Conf., pp. 1337-1344, 1997.

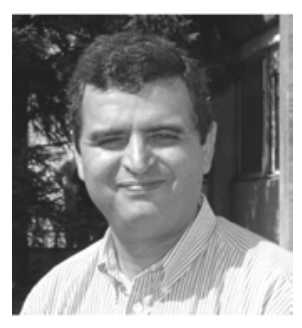

Abdelaziz Hamzaoui was born in Ain Beida in Algeria. He received the Engineer degree in electrical engineering from polytechnic School of Algiers (ENPA), Algeria in 1982, the $D E A$ degree in 1989, and the PHD degree from Reims University of Champagne-Ardenne in 1992, both in electrical engineering. Since October 1992, he has been associate professor with the mechanical engineering department of IUT of Troyes, Reims University, where he is currently the head of the department. His research interests include intelligent control, fuzzy control, and robust adaptive control. Dr. Abdelaziz Hamzaoui is member of IEEE Neural Networks and IEEE Control Systems Societies. He is also member of the IFAC technical committee on Cognition and Control.

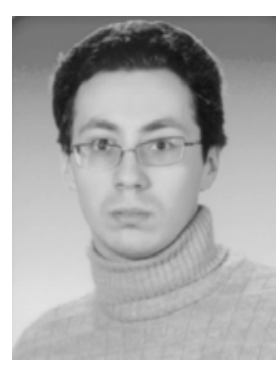

Pavel S. Ustinov was entered the Orel State Technical University (OrelSTU), Orel, Russia, in 1999. He is a graduate student. P.S. Ustinov takes part in the work of scientific group of the Department of Design and Technology of Electronic and Computer Systems. His current research interests include nonlinear dynamics, symbolic dynamics and chaos theory.

Andrey $P$. Sholonik was graduated from the Orel State Technical University (OrelSTU) in 1998. He received the Ph.D. degree in electrical engineering from the Orel state technical university, Orel, Russia, in 2003. A.P. Sholonik is currently an Assistant Professor with the OrelSTU, the Department of Design and Technology of Electronic and Computer Systems, and one of the programming engineers of JSC "Proton-Electrotex". His current research interests include nonlinear dynamics and power electronics.

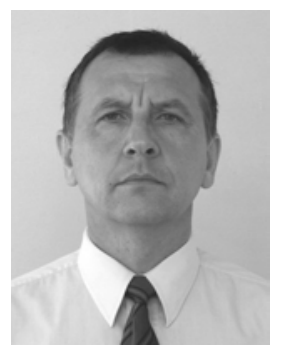

Yury V. Kolokolov was graduated from the Tomsk Politechnical Institute (TPI), Tomsk, Russia, and received the Ph.D. degree in electrical engineering from this institute, in 1972 and 1978, respectively. He received the Doctor of Science (technology) degree in electrical engineering from the Tomsk University of Control and Radioelectronics (TUCR), Tomsk, Russia, in 1990. Yu.V. Kolokolov is currently a Professor with the Orel State Technical University (OrelSTU), a Head of the Department of Design and Technology of Electronic and Computer Systems. His current research interests include system analysis and pulse system nonlinear dynamics. 